\title{
Time domain analysis of a wideband common-mode suppression filter for bent interconnects
}

\author{
C. Gazda ${ }^{1}$, D. Vande Ginste ${ }^{1}$, H. Rogier ${ }^{1}$, D. De Zutter ${ }^{1}$, and R.-B. Wu ${ }^{2}$ \\ ${ }^{1}$ Electromagnetics Group, Department of Information Technology, Ghent University \\ Sint-Pietersnieuwstraat 41, 9000 B-Gent, Belgium \\ E-mail: celina.gazda@intec.UGent.be, Tel.: +32 92643323 \\ ${ }^{2}$ Department of Electrical Engineering and Graduate Institute of Communication Engineering \\ National Taiwan University, Taipei 106, Taiwan
}

\begin{abstract}
A technique to efficiently reduce differential-to-common mode conversion occuring at a bend discontinuity in coupled microstrip lines is investigated. Total signal integrity in the interconnect structure has been improved by minimizing the differential reflection coefficient and insertion loss simultaneously. This is achieved by changing the geometry of the microstrip lines via a tapering section to tightly or very tightly coupled ones in the area of the bend. The new design was manufactured, measured, and validated by means of time-domain analysis, providing very satisfactory results.
\end{abstract}

\section{Introduction}

Differential signaling is a popular way to transmit signals across circuit boards because of its high electromagnetic immunity against conducted and radiated interfering signals [1]. However, when applied, it is important to keep the differentialto-common mode conversion level low, as this phenomenon has a strong influence on the signal integrity [1-5]. The emergence of new technologies, leading to high bit rates, short rise times and small noise margins, makes this requirement even more stringent. Unfortunately, conversion easily occurs, due to signal trace length mismatch [6] or asymmetric discontinuities in the circuit layout, and in particular at a bend of coupled microstrip lines $[2,4,5]$. A reduction of the unwanted common-mode noise can be obtained by applying filtering structures $[4,5]$ or by adding extra compensating capacitance [2].

In [5], a new approach was presented to reduce conversion noise caused by a bend discontinuity over a large frequency range (from DC to $6 \mathrm{GHz}$ ), while maintaining low reflection and insertion loss. Whereas in [5] the focus was on frequencydomain S-parameters simulations and circuit modeling, here, we investigate the behavior of improved bends in time domain by means of measurements. Such time domain analysis provides valuable signal integrity related data for circuit designers.

\section{Problem outline}

When there is no discontinuity in a pair of coupled lines, conversion between differential and common mode does not occur, as long as the interconnect structure remains symmetrical everywhere. In practice, however, it is impossible to avoid discontinuities (such as bends) in the layout on modern circuit boards designed for high frequency systems. Here we investigate a circuit that consists of a classic pair of coupled microstrip lines with a $90^{\circ}$ bend (Fig. 1). The line width is $w=1.8 \mathrm{~mm}$, the complete length is twice $l=50 \mathrm{~mm}$, and the spacing between the lines is $s=0.7 \mathrm{~mm}$ (cross-section $\mathrm{A}$ ). The thickness and conductivity of the microstrips are $t=35 \mu \mathrm{m}$ and $\sigma=4.1 \cdot 10^{7} \mathrm{~S} / \mathrm{m}$, respectively, where the latter is the value for the gold used to plate the copper lines. Note that the structure is symmetrical with respect to the interior bisector. A Rogers RO4350B substrate was chosen $\left(\epsilon_{r}=3.66, \tan \delta=0.003\right.$, $h=1.524 \mathrm{~mm}$ ), because of its low losses and reliability, allowing to perform high-frequency reproducible measurements.

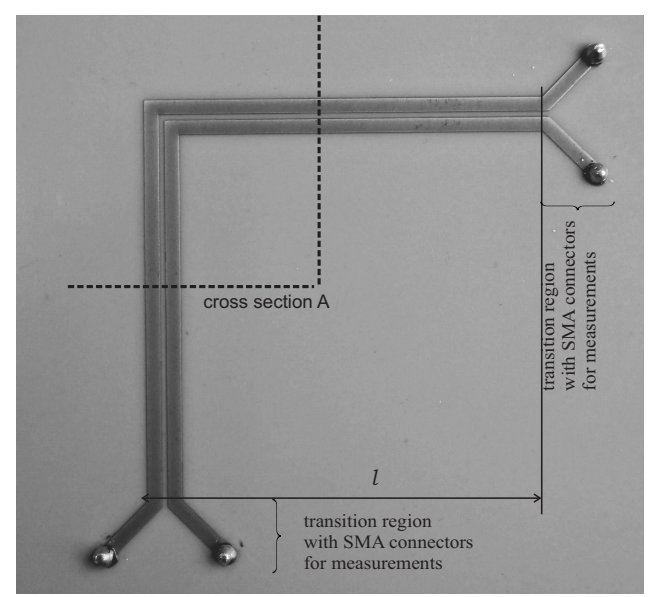

Figure 1: $90^{\circ}$ bend in a classic pair of coupled microstrip lines.

Due to the existence of the bend, the circuit becomes asymmetrical and the lengths of the two parallel microstrip lines are no longer equal, which skews the transmitted signals in time. This introduced delay skew is the main source of differentialto-common mode conversion, causing unwanted noise. A second important aspect concerning mode conversion is found in the values of the differential and common mode characteristic impedances. The structure of Fig. 1 was designed to be matched for differential signals, so the characteristic impedance for the differential mode $Z_{0 d}$ is close to $50 \Omega$, i.e. $50.42 \Omega$, whereas for the common mode the characteristic impedance $Z_{0 c}$ is $87.47 \Omega$. Although that results in $37.05 \Omega$ of difference between these impedances, when the asymmetric bend discontinuity is present in the structure this difference seems to be too small to prevent the differential mode from converting to the common mode.

\section{Improved layout}

To alleviate the mode conversion problem, the structure needs to be modified such that the length difference between the microstrips is smaller and such that the difference in impedance values between the modes is larger. At the same time, the differential mode impedance should remain close to $50 \Omega$ along the length of the structure, to avoid mismatch. Two versions of an improved structure, here called "tightly coupled" and "very tightly coupled", have been investigated (Fig. 2). In both cases, the classic microstrip lines (with cross-section A) are connected 
with tightly or very tightly coupled lines (with cross-section B or $\mathrm{C}$, respectively) through a tapering section with a length $l_{t}$. Table 1 summarizes the dimensions for both cases. Obviously, tightly or very tightly coupled microstrip lines are applied only in the area of the bend, due to their higher losses. In this way, and also by using a low-loss substrate in the design, we avoid high losses in the overall interconnect.

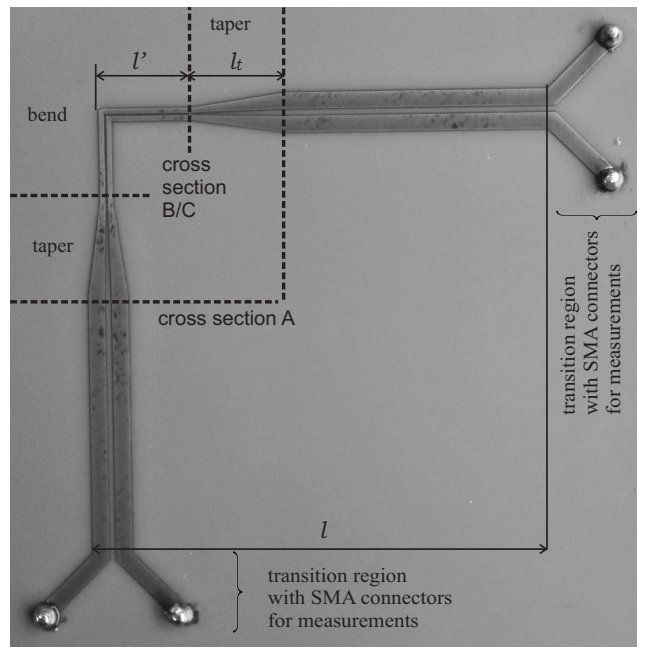

Figure 2: Proposed improved coupled microstrip lines bend. Cross-section A: classic microstrip lines; cross-section B or C: tightly or very tightly coupled microstrip lines.

\begin{tabular}{|c|c|}
\hline parameter & value \\
\hline & tapering section \\
\hline$l_{t}$ & $10 \mathrm{~mm}$ \\
\hline \multicolumn{2}{|c|}{ tightly coupled (cross-section $\mathrm{B}$ ) } \\
\hline$w$ & $600 \mu \mathrm{m}$ \\
\hline$s$ & $200 \mu \mathrm{m}$ \\
\hline$l$ & $10 \mathrm{~mm}$ \\
\hline very tightly coupled $($ cross-section $\mathrm{C})$ \\
\hline$w$ & $300 \mu \mathrm{m}$ \\
\hline$s$ & $150 \mu \mathrm{m}$ \\
\hline$l$ & $10 \mathrm{~mm}$ \\
\hline
\end{tabular}

Table 1: Geometric parameters

Compared to Fig. 1, the length difference between the two microstrips of Fig. 2 is significantly reduced, yielding a reduction in differential-to-common mode conversion. Moreover, in both versions of the structure a larger difference in characteristic impedance between the two modes is obtained. For the new layout, in the region of the tightly coupled bend, the characteristic impedance for the differential mode $Z_{0 d}$ equals $49.72 \Omega$, compared to $Z_{0 c}=159.65 \Omega$, for the common mode, resulting in $109.93 \Omega$ of difference. In the region of the very tightly coupled tapered bend, $Z_{0 d}$ equals $50.08 \Omega$ and $Z_{0 c}$ is equal to $204.98 \Omega$, resulting in $154.90 \Omega$ of difference. Thus, the modifications cause higher coupling between the lines, acting as a natural common mode suppression filter, as such improving the signal integrity behavior of the bend. The value of the parameter $l^{\prime}$ is a trade-off between sufficient length of the common mode suppression filter, a compact size of the complete interconnect, and efficient separation of the tapers, which helps to avoid direct field mutual coupling.

\section{Time domain results}

First, a TDR (Time Domain Reflectometry) analysis was performed. A ramped step signal, going from $0 \mathrm{~V}$ to $1 \mathrm{~V}$ with a rise time of $t_{r}=30 \mathrm{ps}$, was injected into the SMA of the outer (longer) line. Simultaneously, a ramped step signal going from $0 \mathrm{~V}$ down to $-1 \mathrm{~V}$ with a fall time of $t_{f}=30 \mathrm{ps}$, was injected into the SMA of the inner (shorter) line. As such, a perfect differential input signal was applied. The output ports, i.e. the SMAs at the receiving ends, remained open. In Fig. 3, the reflected voltages at the input ports for the three proposed bends are observed. As shown in Fig. 3, the introduced geome-

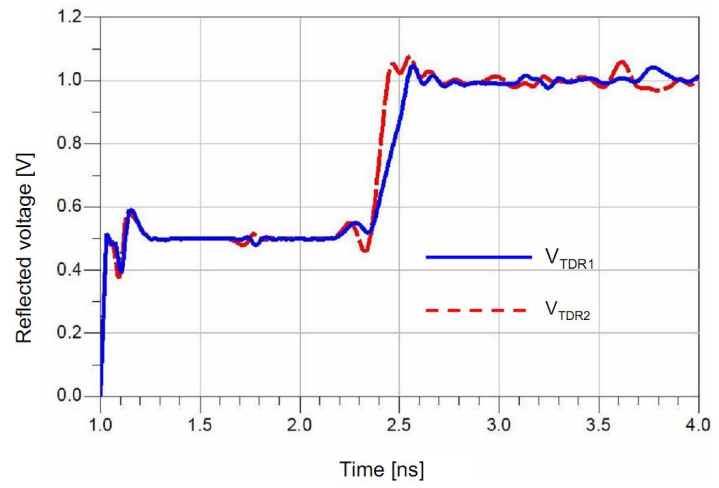

(a) Classic bend

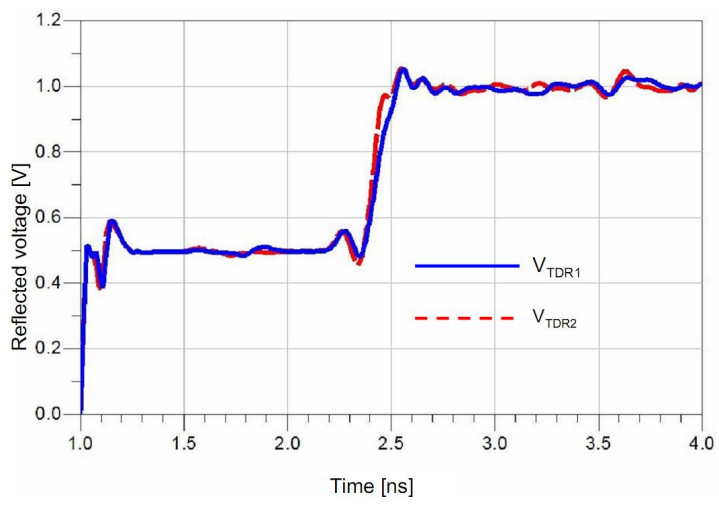

(b) Tightly coupled bend

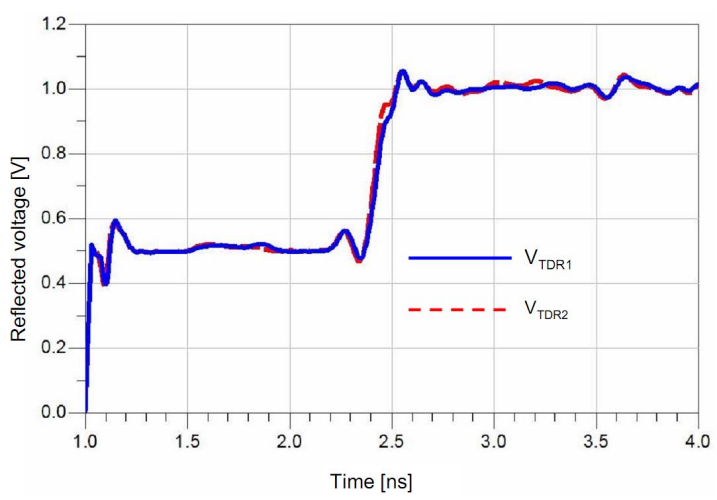

(c) Very tightly coupled bend

Figure 3: TDR results of the three described structures.

try modification significantly reduces the skew in time between 
the propagating signals. In case of the classic bend (Fig. 3(a)), the two voltages are skewed by 48.5 ps (measured when the reflected signals reach $1 \mathrm{~V}$, corresponding to the reflection at the open output ports), whereas for the tightly coupled bend this skew is equal to $6.5 \mathrm{ps}$. Additional improvement is visible for the very tightly coupled bend, as the time skew for this case is only 3.5 ps. In Fig. 3 we also notice the presence of the transition regions with SMA connectors, as well as a small increase of the reflected signals caused by the tapers and the bend discontinuity.

Second, a TDT (Time Domain Transmissometry) analysis was conducted. The input signals were the same as in the TDR setup, but now the receiving ends of the lines were matched to $50 \Omega$ loads. As presented in Fig. 4, by applying our proposed solution, a large reduction of common mode noise was obtained. In case of the tightly coupled bend, the reduction in common mode noise is equal to $56.6 \%$, whereas for the very tightly coupled bend we obtain a reduction of $72.1 \%$. When the input signal was changed to a sequence of pseudo-random bits (PRBS) with a bit rate of $10 \mathrm{Gbps}$, and a rise/fall time $t_{r / f}=30 \mathrm{ps, \text {we }}$ obtained eye diagrams as depicted in Fig. 5. It is clear that the new structures greatly improve signal integrity at the receiving ends, resulting in a larger eye opening.

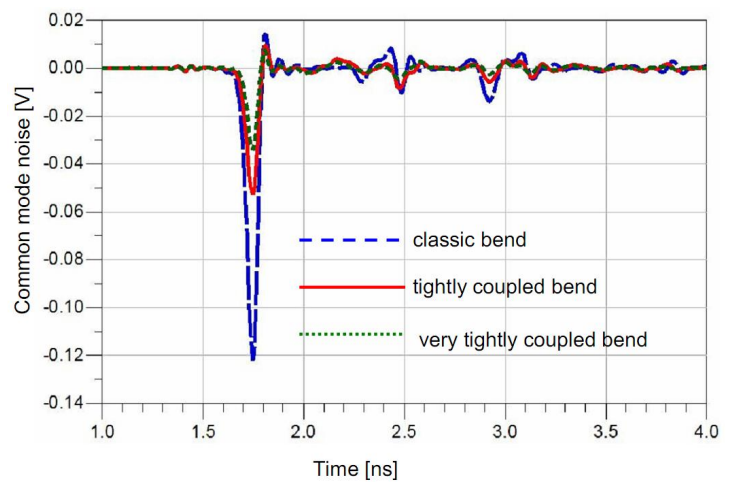

Figure 4: Common mode noise at the receiving port for the three bends.

Third, we introduced time skew between the coupled microstrips. In Figs. 6(a) and 6(b), two examples are shown, where the ramped step input signal at the inner (shorter) line was delayed by $10 \mathrm{ps}$ and $20 \mathrm{ps}$, respectively, i.e. $33 \%$ and $66 \%$ of the rise time. By comparing Figs. 4 and 6(a) we observe that the common mode noise is reduced for all three cases. For the very tightly coupled bend, the noise becomes even minimal. However, for the other case (Fig. 6(b)), we observe that the performance of the classic bend still improves but that the behavior of the new bends gets worse, as these structures got overcompensated. If a time delay was applied to the outer line, in all cases we observed deterioration of the results.

It is understandable that by applying a well-chosen skew value, the signal integrity behavior of each bend can be improved. In practice, however, differential drivers do not produce perfect differential signals or signals with a constant time skew. Typically, jitter with a random distribution is present. Therefore, the results of Fig. 5 were re-evaluated in Fig. 7, where

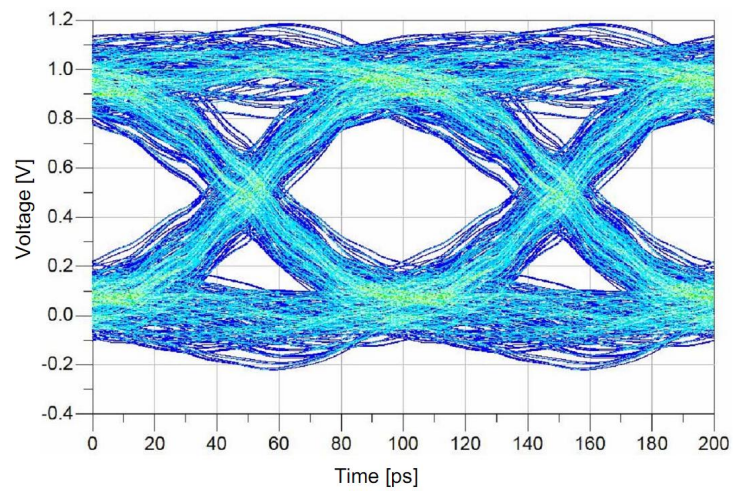

(a) Classic bend

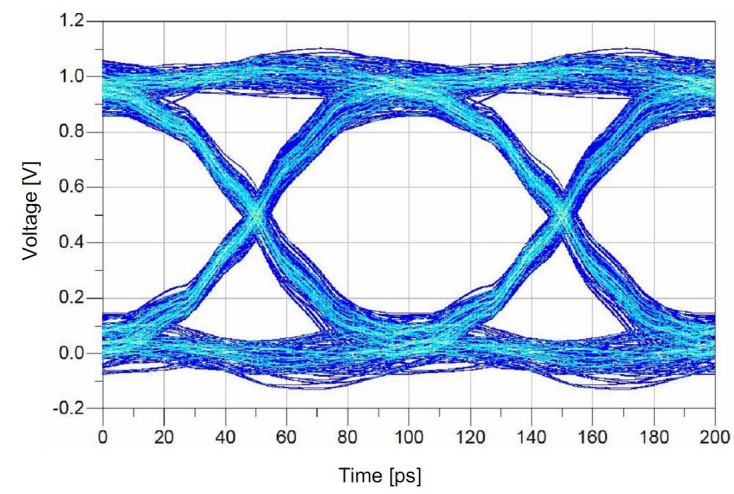

(b) Tightly coupled bend

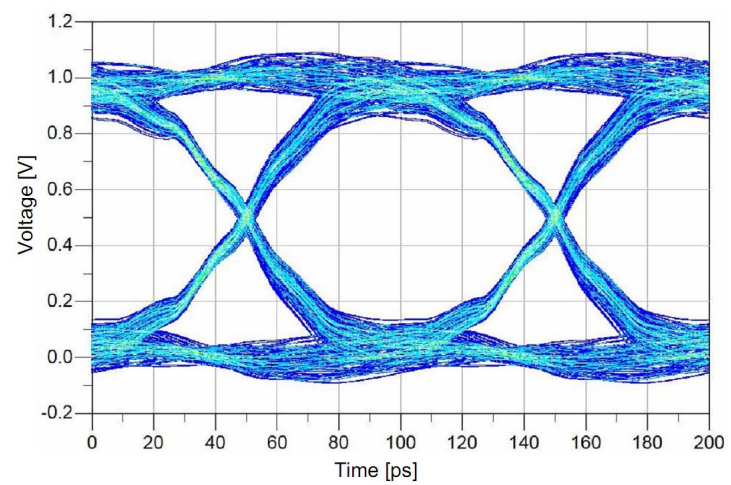

(c) Very tightly coupled bend

Figure 5: Eye diagrams for the three bends when a perfect differential signal is injected.

now an additional random jitter with standard deviation of $20 \mathrm{ps}$ has been added to the PRBS. It is clearly visible that even under this rather harsh conditions the new layouts bring great improvement, resulting in a larger eye opening (concerning both the width and the height).

\section{Conclusions}

The issue of mode conversion occurring at the bend discontinuity in a pair of coupled microstrip lines has been studied. New routing layouts, consisting of a tightly or a very tightly coupled tapered bend were designed, manufactured, and measured to analyze their behavior in time domain. When applying perfect differential signals, experimental validation of the pro- 


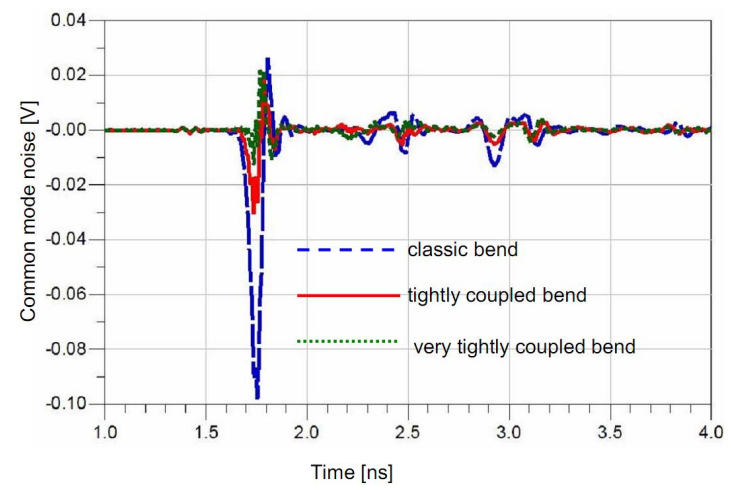

(a) Additional 10 ps time skew.

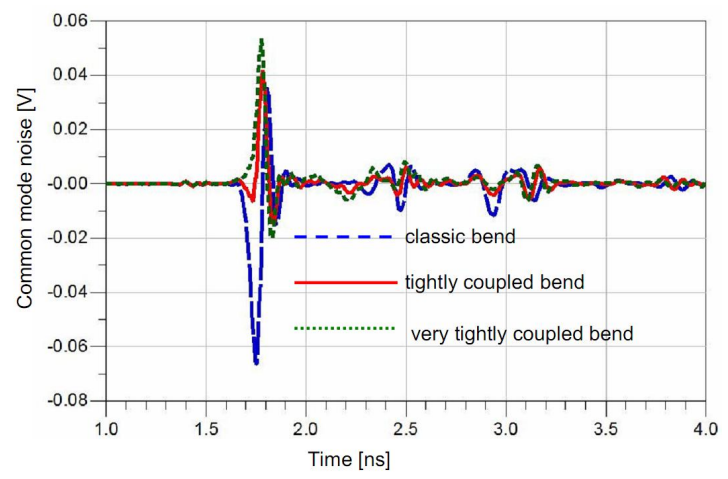

(b) Additional 20 ps time skew.

Figure 6: Common mode noise at the receiving port with additional time skew.

posed structure proves that differential-to-common mode conversion is significantly suppressed. This is thanks to the reduction of the length difference between the traces and the larger difference in impedance for the differential and common mode. Moreover, even in the presence of the time skew and jitter in the input signal, the new bends largely outperform the classic one.

\section{References}

[1] S. Connor, B. Archambeault, and M. Mondal, "The impact of common mode currents on signal integrity and EMI in high-speed differential data links," in IEEE Int. Symp. on EMC, Aug. 2008, pp. 680-684.

[2] G.-H. Shiue, W.-D. Guo, C.-M. Lin, and R.-B. Wu, "Noise reduction using compensation capacitance for bend discontinuities of differential transmission lines," IEEE Trans. Adv.Pack., vol. 29, no. 3, pp. 560-569, Aug. 2006.

[3] B. Archambeault, J. Diepenbrock, and S. Connor, "EMI emissions from mismatches in high speed differential signal traces and cables," in IEEE Int. Symp. EMC, 2007, pp. $1-6$.

[4] F. De Paulis, A. Orlandi, L. Raimondo, B. Archambeault, and S. Connor, "Common mode filtering performances of planar EBG structures," in IEEE International Symposium on Electromagnetic Compatibility, Aug. 2009, pp. 86-90.

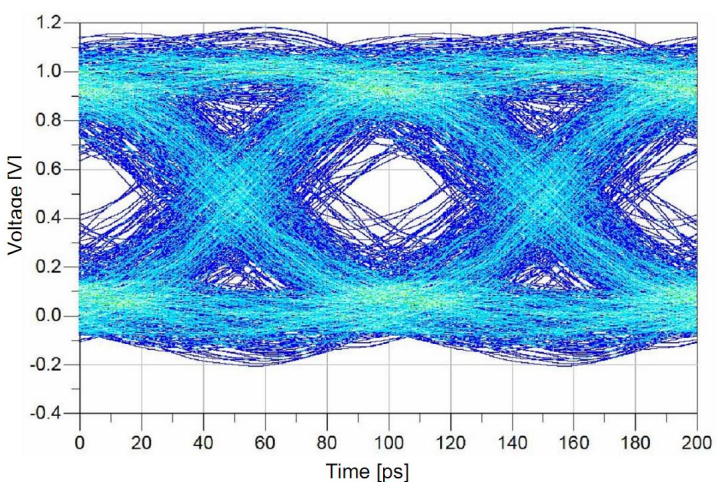

(a) Classic bend

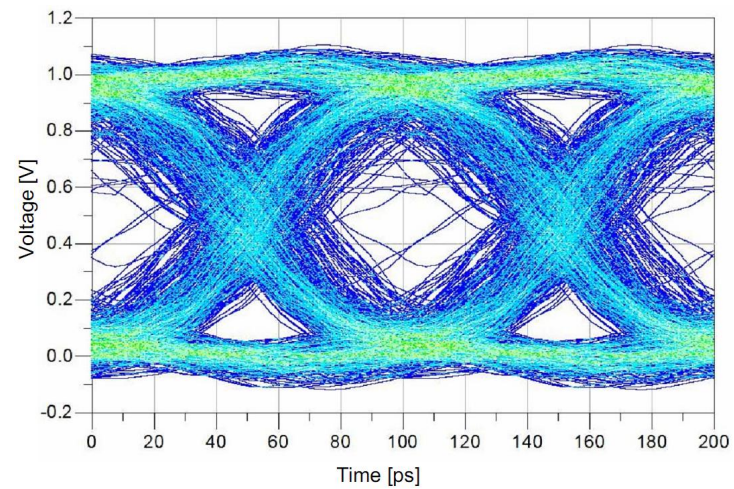

(b) Tightly coupled bend

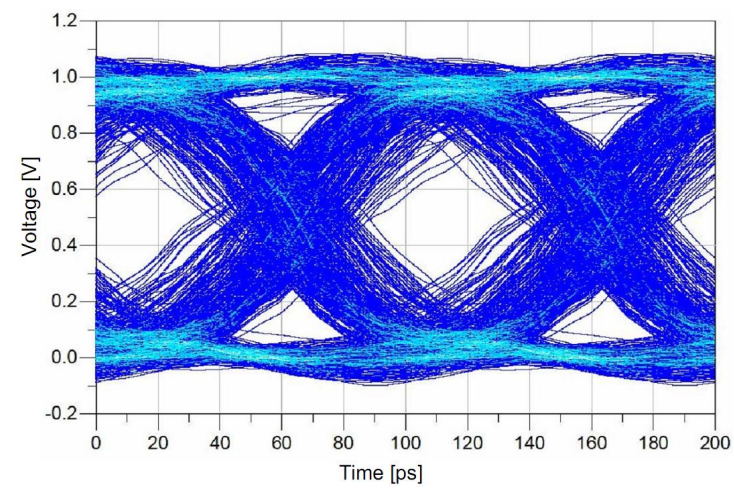

(c) Very tightly coupled bend

Figure 7: Eye diagrams for the three bends when 20 ps jitter is introduced.

[5] C. Gazda, D. Vande Ginste, H. Rogier, R.-B. Wu, and D. De Zutter, "A wideband common-mode suppression filter for bend discontinuities in differential signaling using tightly coupled microstrips," IEEE Trans. Adv. Pack., vol. 33, no. 4, pp. $969-978,2010$.

[6] J. Knighten, N. Smith, J. DiBene, and L. Hoeft, "EMI common-mode current dependence on delay skew imbalance in high speed differential transmission lines operating at 1 gigabit/second data rates," in IEEE Proc. of 1st Int. Symp. Quality Electr. Design, 2000, pp. 309-313. 